\title{
Esbozo histórico-conceptual de la psicología social comunicativa, desde un punto de vista comunicativo.
}

\author{
Angélica Bautista López \\ Universidad Autónoma Metropolitana (México) \\ Gustavo Martínez Tejeda \\ Universidad Pedagógica Nacional \\ blal@xanum.uam.mx
}

\section{INTRODUCCIÓN}

Cuando hablamos del desarrollo histórico de la Psicología Social, notamos que existen diferentes versiones al respecto. Se puede considerar a este hecho como parte de su propia crisis. Dicha crisis evidencia el problema que existe con relación a la poca incidencia que se aprecia en la realidad que interesa explicar, a partir de los aportes que surgen dentro de la disciplina. La desconexión entre realidad social y teoría es la muestra evidente de que el desarrollo de la Psicología Social no ha igualado al desarrollo de la sociedad. El objetivo principal de esta disciplina es el de explicar el acontecer de la sociedad. Este acontecer "psicosocial" incluye la manera repensar y de sentir de la sociedad. Cuando esto no se cumple, como es el caso de la Psicología Social de corte individualista, se aprecia en ello una razón histórica.

Cuando se habla de historia generalmente se evoca a un conjunto de hechos y datos cronológicos cuya continuidad temporal, por sí sola, supone la continuidad lógica de una disciplina. Nos referimos entonces, a una presunta continuidad conceptual ${ }^{1}$. Sin embargo, esto no sucede así, ya que el pasado deber ser analizado desde el presente para ubicar en aquel su surgimiento y desarrollo.

Para explicar esto a lo que nos referimos, tomemos como premisa básica que: "la reacción teóricoconceptual de una disciplina cumple el mismo proceso que el de la creación cultural en general". La pluralidad de explicaciones enriquece a la disciplina y permite la creación subsecuente de nuevas interpretaciones, sin embargo, no todas son consensuadas. Tal es el caso de la Psicología Colectiva, que surge en esta pluralidad de explicaciones, pero que al romper con las alternativas teóricas consensuadas, fue desarrollándose en "silencio" marginándosele de la difusión y el reconocimiento profuso.

El interés central del presente trabajo, el desarrollo histórico conceptual de la Psicología Colectiva, analizando a partir del momento actual: de sus planteamientos básicos y de sus temas de interés teórico y social. El desglose histórico que esto supone requiere de un contraste conceptual con el resto de las aproximaciones, básicamente por dos razones:

${ }^{1}$ Para historias de la Psicología Social ver Allport, 1968. 
Primero porque es en este contraste en donde realmente se ubica el desarrollo que nos ha llevado a este presente, y Segundo porque reconstruir críticamente el pasado permite generar aproximaciones más acordes a la realidad social que nos compete. El objetivo básico y final de este trabajo se centra en el proyecto de futuro que se desprende del análisis planteado: Un proyecto de Psicología Colectiva con dos intereses: el teórico, la comunicación colectiva, y el social, la cultura.

\section{El presente de la Psicología Social: Psicología Colectiva}

La Psicología Social en la actualidad contiene un gran número de interpretaciones. Estas tienen, a su vez, un sinnúmero de divergencias y convergencias. La explicación de tal variedad se ubica en la realidad misma. Todas las interpretaciones existentes tratan de develar lo que el sentido común oculta: los procesos que permiten el desarrollo de la sociedad. En su intento de explicación, esta disciplina construye sus objetos de estudio y los transforma al descubrir que requiere de otros más cercanos a la realidad. Los planteamientos de los que se desprenden todas las construcciones teóricas actuales son dos: a saber:

Por un lado tenemos a todas las teorías que ubican su interés en la analogía individuo - sociedad. Decimos analogía porque se trata de un modelo teórico, con su correlato filosófico (dualismo²), que se aproxima a la realidad dividiéndola para su estudio. En este sentido, el modelo pierde lo más relevante de la realidad que es lo que conforma su totalidad.

El énfasis que a lo largo de este siglo, por lo menos a partir de los 20's, se le ha dado al "manejo" y "control"3 hizo que una de las interpretaciones que se desprende de esta primera construcción teórica, la Psicología Social de corte individualista, adquiera una mayor difusión, logrando hasta hace unos años ser llamada la Psicología Social. Digamos que se trataba de la interpretación consensuada hasta ese momento.

Sin embargo, bajo esta construcción teórica, podemos ubicar también a la Psicología Social europea, a la Psicología Social de la Personalidad, a la Psicología Social Marxista, entre otras. Esto es posible debido a que, a partir de dicha polaridad, se han creado teorías que ubican su interés en dos aspectos del mismo problema.

En el primero se ubican las que estudian la interacción individuo - sociedad, ya sea desde la óptica del individuo: Psicología Social individualista; ya sea desde la óptica de la sociedad: psicología social europea.

En el segundo las que centran su interés en el eje de dicha polaridad: el individuo, o mejor dicho, su personalidad, ya sea desde la óptica del individuo libre y determinante de su entorno, como es el caso de las teorías de liderazgo y en general la psicología social de la personalidad; ya sea desde la óptica

${ }^{2}$ Dentro de esta filosofía moderna se encuentra esta versión dualista del mundo, sea entre la razón y la libertad (Kant), sea entre el individuo y la sociedad en general (Garai y Eros, 1978). Su origen se ubica en el iluminismo (siglos XVII y XVIII) y en el arraigo de la filosofía positivista en las Ciencias Sociales.

${ }^{3}$ Existe una explicación evidente a ese énfasis por predecir y controlar, de corte económico - político, pero también existe una explicación de corte más social, ligada al sentido común en la que, los hombres buscan un proyecto de vida a partir de ellos mismos, en donde todo debe ser controlable. Esto puede, a su vez, explicarse por la misma construcción social del individualismo. 
del individuo determinado por sus condiciones sociales, como es el caso de la psicología social marxista.

Por el otro lado tenemos a las aproximaciones teóricas que plantean un ejercicio de interpretación globalizador con respecto a la realidad. Parten de la idea de que la totalidad que integra la realidad, a pesar de que no puede ser conocida en su marco real, no debe ser reducida a elementos. Aquí es precisamente en donde se ubica la psicología colectiva. En ella se incluyen teorías interesadas en el estudio de las colectividades, el estudio de las masas, multitudes, etc.

El punto de vista del que parte este trabajo está ubicado en esta psicología colectiva. Para comprenderlo es necesario explicitar su fundamentación teórica y sus temas de interés, ya que es a partir de estos que se plantea la reflexión con respecto a su desarrollo histórico.

\section{El fundamento de la psicología colectiva: la intersubjetividad}

Decíamos que la finalidad de la psicología social es la de explicar la realidad social, y si la cualidad más relevante de dicha realidad social es su capacidad de transformación constante, resulta comprensible el interés que las diferentes aproximaciones han manifestado por el estudio de la interacción. La relación entre las personas es, evidentemente, el punto ubicable de dicha transformación. Con todo y esto, no significa que la interacción sea el constructo teórico más cercano a la transformación social.

Existen diversos argumentos que apoyan esta aseveración. La realidad es construida socialmente, y en esta construcción se encuentra un elemento sumamente importante, este es el elemento simbólico. Cuando se hace referencia a un proceso de transformación social, se está incluyendo la construcción de lo imaginario, de todo aquello que rebasa la situación particular de los actores de cualquier interacción.

La alteridad es todo ese cúmulo de elementos imaginarios que significan a los actores involucrados en dicha interacción. Incluir estos elementos en un constructo teórico nos lleva al planteamiento de la intersubjetividad (Fernández, 1987), Lo relevante de la realidad social, dentro de este punto de vista se ubica entre los individuos. Sus características personales, por lo tanto, son un constructo social y son también la manifestación temporal de este proceso comunicativo.

Analizar dichas características personales (personalidad) o las acciones específicas que suceden en su manifestación temporal (Psicología Individual), es un intento a-histórico y a-ideológico de explicar nuestra realidad.

\section{El tema de interés teórico de la Psicología Colectiva}

Para la Psicología Social en general, sin importar la tradición a la que se circunscriba, resulta evidente que el tema central en la actualidad es la comunicación. Desde la atracción interpersonal, el conflicto social, la influencia social, la atribución social o el desarrollo cultural, todas "las psicologías sociales" han llegado a un punto en el que resalta la necesidad de estudiar este tema deviene de su fundamento teórico básico. La intersubjetividad es en realidad, un constructo procesual de la comunicación. En este sentido, ubica el origen de su desarrollo en Mead (1930), ya que este autor define este proceso intersubjetivo de comunicación cuando afirma que: 
“...lo esencial pala la comunicación es que el símbolo despierte en la persona de uno lo que despierta en el otro. Tiene que tener esa clase de universalidad para cualquier persona que se encuentre en la misma situación" (Mead, 1930)

Es a partir del desarrollo de este planteamiento, encontramos que la comunicación intersubjetiva permite explicar más globalmente nuestra realidad social.

\section{El tema y objeto social relevante para la psicología colectiva: La Cultura}

Ya en la introducción se había anotado la distinción entre "las psicologías sociales" interesadas en algunos aspectos de la realidad, en contraposición a la psicología colectiva, interesada en la realidad. Para esta, el tema central de la realidad es la cultura. En este sentido, los análisis pueden incluir los "datos más diversos, ya que lo importante es ubicarlos en su marco histórico e ideológico, y esto no se logra a partir de la teorización que actualmente se desarrolla sobre comunicación e intersubjetividad.

El estudio de la cultura se contrapone a un orden de ideas de tipo "metodológico-tradicional", ya que rescata lo que da el sentido a las situaciones, a las vivencias de las ideas, a los sentires y en general a las manifestaciones y creaciones culturales. Para ello se requiere generar una propuesta conceptual-metodológica (Haberlas, 1982).

\section{El pasado en psicología social: una reinterpretación Histórico-Conceptual}

Hasta este momento se han planteado las líneas generales que guían este trabajo. Se trata de ubicar los momentos histórico-conceptuales que han marcado el desarrollo de la psicología colectiva ${ }^{4}$, desde los planteamientos referidos con anterioridad. El inicio de esta narración se ubica en la filosofía, con el surgimiento y desarrollo de la concepción dualista.

\section{De la polaridad individuo-sociedad a la interacción}

A partir de la postura dualista se generaron un sinfín de explicaciones en torno al problema del hombre y del conocimiento que este logra de su mundo. Con relación a esto, la Psicología Social tiene ante sí una tarea doble: por un lado, dar explicación a las razones que llevan a "hacer" a los hombres. Esta tarea surge con el planteamiento del concepto "libertad o libre albedrío" dentro de la filosofía. Y por el otro, a la preocupación, filosófica también, de dar cuenta de las condiciones sociales que, contrariamente llevaban a la opresión de los hombres.

La polaridad individuo-sociedad era, entonces, un constructo teórico que requería un desarrollo en doble sentido: Uno al referirnos a como el individuo "crea, hace y logra" en la sociedad y otro, al plantear como la sociedad "crea, hace y logra" al individuo.

\footnotetext{
${ }^{4}$ Se entiende por momento histórico-conceptual al surgimiento de una nueva interpretación, aún y cuando los datos cronológicos sean disparados.
} 
En los siglos XVII y XVIII surgen las bases filosóficas de esta polaridad. El iluminismo pretende negar la contradicción entre los dos puntos de vista al plantear un equilibrio entre la libertad social y la libertad individual. A partir de aquí se sientan las bases del desarrollo de la psicología Social Norteamericana. La necesidad de explicar, y sobre todo convencer de este equilibrio es retomada., A raíz de esto, el desarrollo de este tipo de Psicología se centra en el individuo.

El individualismo que surge en este tiempo permea el desarrollo de las sociedades. De esta manera la Psicología Social Norteamericana, al seguir este desarrollo ${ }^{5}$, se aleja de las preocupaciones sociales y tiende a desarrollar explicaciones y paradigmas muy cercanos a la Psicología Individual ${ }^{6}$. Para el dualismo, existe una realidad dividida en dos instancias que se relacionan de alguna manera. De aquí que se afirme como objeto de estudio de la Psicología Social a la interacción.

Tenemos entonces un sinfín de definiciones de interacción, todas las que conlleva el surgimiento de las diferentes aproximaciones de la Psicología Social. Dentro de estas la que ocupa un espacio teórico menor es la Psicología Social Norteamericana. Su desarrollo ha sido escaso, pero su difusión y dominio son tales que, aún ahora, para muchos interesados en estas cuestiones es la Psicología Social.

A partir de los años 20's se puede afirmar que, lo que surgió dos siglos atrás como una versión adelantada de la filosofía, léase filosofía positivista, se instauró como una ideología individualista ante la cual los científicos, en su mayoría norteamericanos generaron una serie de planteamientos acordes a este sentido común vigente. La mayoría de estos de tipo conductual. Un ejemplo extremo de la visión social que esta aproximación entiende es el caso de teorías como la facilitación social. Aquí, lo social se incluye con la presencia física de otra. Esta psicología Social supone una continuidad de especies que sería objeto de otra discusión ${ }^{7}$. De esta manera, sus estudios infrahumanos "permiten explicar la conducta del hombre", veamos una cita ilustrativa al respecto:

“...si analizamos las reacciones de la rata, al 'girar hacia la izquierda del tablero en $T$ ', cuando se encuentra otra rata en el brazo derecho del tablero, entonces realizamos una función de psicólogo social" (Zajonc, s/f, p.8).

En términos generales, esta psicología social define la interacción bajo los mismos planteamientos que la psicología individual, retomando el paradigma $E-R$ y agregando a las explicaciones anteriores el aspecto social, en términos de otro organismo. El paradigma $\mathrm{E}-\mathrm{R}$ es reformado en este sentido, sin una revisión crítica. Por esta razón se asume como ciencia natural y ante esto, asume también un enfoque metodológico que "observa, verifica, comprueba y predice".

Todo lo observable, verificable, comprobable y predecible forma parte sólo de la manifestación externa y conductual de la realidad. De esta forma, sólo describe un elemento de la realidad. Al no incluir la totalidad, su capacidad de explicar la realidad es inexistente. Sus temas ampliamente

${ }^{5}$ Es innegable que este alejamiento supuso también la existencia de factores histórico - políticos determinantes, y sobre todo, de corte económico.

${ }^{6}$ La Psicología Individual se ampara en un análisis intraindividual, en donde lo externo queda totalmente excluido (Doise, 1983).

7 Dicha continuidad, si se tomara como argumento teórico, sería una continuidad evolutiva que debería retomar, también, una continuidad genético-evolutiva en el terreno de las emociones y de los afectos. 
difundidos carecen de desarrollo teórico y sólo describen elementos faltos de relación tales como: las actitudes, la atracción interpersonal, el liderazgo, etc.

\section{De la interacción a la interacción social}

A pesar de que para la psicología social norteamericana, su objeto de estudio sea la interacción y, según los apellidos que se le agregan a las explicaciones individualistas, se plantee como interacción social la realidad es otra. Sus explicaciones se ubican en el lado observable de las cosas y su inferencia (ineludible) se ubica al interior de cada organismo, de una manera a-histórica y aideológica. Este tipo de interacción es rechazada por otra aproximación que surge bajo el mismo techo que la anterior.

En la discusión constante de sus trabajos, algunos teóricos (en los 40's) comienzan a esbozar un "nuevo" objeto de estudio: la interacción social. Autores como Heider, Festinger, Asch, Sherif y Sherif, Lewin, entre otros, discurren en un tipo diferente de concepción ${ }^{8}$. Este énfasis es apreciado vivamente en el interaccionismo simbólico de George $\mathrm{H}$. Mead. En este trabajo se reconoce una interacción que ha dejado de preocuparse por el individuo o por el medio. Surge entonces el interés por el vínculo entre ambos.

Este "nuevo" objeto de estudio ubica su interés en los elementos simbólicos de las relaciones. Aquí no es relevante la manifestación conductual sino su contraparte oculta: la conciencia. Es en realidad una ruptura conceptual de grandes proporciones, pero que en un primer momento subsiste con los planteamientos iniciales. De esta manera mantiene incólume su forma de abordar la realidad. La metodología sigue siendo enfocada de la misma manera. Posteriormente, a la luz de la actual crisis de las ciencias sociales, esta ruptura conceptual es reflexionada, analizada y desarrollada en lo que conocemos como Psicología Social Europea (Tajfel, 1972).

Surge entonces una necesidad por explicitar esta ruptura, no sólo en el plano teórico, como es el caso de los modelos de interacción de Moscovici (1972), sino en el aspecto conceptual metodológico, como sucede en el trabajo sobre los niveles de análisis de Doise (1983).

Los temas de interés de esta Psicología Social son, entre otros, la categorización social, la influencia social y la representación social ${ }^{9}$ y buscan dar cuenta de la realidad en términos globales.

Así como se aprecia que la psicología Social Norteamericana ha llegado ya a su límite de desarrollo conceptual, se aprecia que la Psicología Social europea aún no alcanza su verdadero desarrollo. Esto se vislumbra en la necesidad de crear un marco conceptual-metodológico en torno a esto: básicamente el interés por una metodología de corte hermenéutico, pero en este terreno aún queda mucho por hacer.

\footnotetext{
${ }^{8}$ SE hace referencia a trabajos como la Teoría de la Atribución de Heider (1946), el cognoscitivismo de Festinger (1957), la percepción social de Sherif y Sherif (1969), el gestaltismo de Asch (1952) y la teoría de campo de Lewin (1951).

${ }^{9}$ Tajfel, 1981 y Moscovici, 1981; 1961 respectivamente. Además, en esta Psicología Social se ubican autores como Billig, Doise, Deschamps y Mugny, entre otros.
} 


\section{De la interacción social al interaccionismo}

A partir de la conceptualización de interacción social surgió, como lo vimos líneas arriba, la aproximación de la Psicología Social europea, que centra su interés en la relación individuo-sociedad. Pero acorde con esta idea global de lo social existe otra aproximación que integra esta visión interactiva a su propio análisis del individuo. Con el énfasis centrado en el individuo y desde el punto de vista opuesto al de la Psicología Social Norteamericana, surge a finales del siglo pasado, con autores como Cabriola y Plejánov (Munné, 1982), una psicología social derivada de los planteamientos creados por Marx (1844), la psicología social marxista se interesa en conocer las cuerdas que accionan, en el ámbito simbólico, a los individuos bajo una determinada sociedad. A partir de los constructos teóricos marxistas: alienación y propiedad privada, intenta deslindar las razones y las formas de convivencia al interior de una sociedad capitalista. Su interés en la alienación estriba en poder explicar a un individuo reproductor de una sociedad y alejado del trabajo, en general y del productor de su trabajo y de su actividad misma, en particular.

Dicha alienación explica la falta de sentido de la vida, que surge en las sociedades industrializadas. Existe, pues, una alienación objetiva pero, a la par, se teoriza una alienación simbólica subjetiva que hace converger los elementos externos con los internos. A pesar de centrarse en un estudio de personalidades, su desarrollo enfoca la totalidad de la realidad. A lo largo de este siglo han surgido continuadamente trabajos que se ubican en esta aproximación ${ }^{10}$ y en este momento se aprecia unas preocupación semejante a la de la psicología social europea: la reiterada apreciación de que las relaciones individuo - sociedad son interactivas.

A partir de esta reflexión se aprecia la cercanía entre Marx (1845) y Mead (1930) en el terreno de la formación de la conciencia. Esta similitud se ubica en el sentido de que la psicología social marxista utiliza un marco intersubjetivo para explicar la intraindividualidad, en tanto que la aproximación que se desprende de Mead integra en su enfoque intersubjetivo una realidad globalizada.

\section{Del interaccionismo simbólico a la intersubjetividad}

A fines del siglo pasado y principios del presente, mientras las aproximaciones antes referidas buscaban su ubicación al interior de la polaridad individuo - sociedad, surge a la par otra aproximación. En esta, el supuesto básico es el de una realidad integral que debe ser analizada en este sentido. Se trata de la psicología colectiva, cuyos gestores se interesaron por explicar a las masas y las colectividades ${ }^{11}$, en un período que va de 1890 a la década de los 20 's.

El estudio de las masas y las colectividades supone una entidad diferente a la estudiada por el resto de las aproximaciones. En esta psicología no se enfatiza ni el estudio del individuo ni el del medio ambiente ni el de la personalidad. Por el contrario, se explica una realidad creada intersubjetivamente que es cualitativamente distinta a la de los individuos.

${ }^{10}$ En esta aproximación se ubican los trabajos de Lucáks; la escuela de Frankfurt en los 20's, con Adorno, Horkheimer y Marcase; Reich en los 30's; Mannheim y Lefebvre en los 40's; Lévi-Strauss y su estructuralismo antropológico en los 50's y Godmann, Heller y Haberlas, de los 60's en adelante.

${ }^{11}$ Con obras como la de Lebon, Sighele, Rossi y Freíd en cuanto al estudio de las masas y las de Wundt y Durkheim con relación a las colectividades. 
Este argumento permite ubicar los trabajos de masas y colectividades bajo la misma aproximación, a pesar de las diferencias que existen entre ambos constructos. Tanto las masas como las colectividades son entendidas aquí como el sujeto colectivo. En la primera conceptualización se explica una irrupción directa en la realidad social (masas) en tanto que, en la segunda aparece y se aprecia por medio de sus objetivaciones.

Como se ha referido líneas arriba, esta psicología colectiva surge a principios de siglo, sin embargo, a partir de los 20's (época en la que el resto de las aproximaciones se desarrollan con mayor viveza), la psicología colectiva se disipa. La razón de este hecho no es totalmente clara, sin embargo, parece que el interés por la realidad social totalizada negaba por consecuencia lógica la posibilidad de controlar y predecir la realidad. El afán de poder llevó a negar una aproximación que de suyo merecía el título de la psicología social.

Aún cuando los tiempos cronológicos parezcan desfasados en términos de las tendencias histórico conceptuales, es a partir de la mitad del siglo XX que se puede hablar de una recuperación de esta psicología colectiva ${ }^{12}$, lo que en términos de la disciplina en general nos lleva a hablar de un giro conceptual que va de la interacción social y del interaccionismo simbólico a la intersubjetividad. Dicha recuperación se inicia con los trabajos de la psicología social europea (Moscovici, 1981) y con los planteamientos de los interaccionistas simbólicos (Berger \& Luckman, 1983).

Para la psicología colectiva, el problema metodológico está íntimamente ligado a su postura teórica: si la realidad conforma un todo diferente a los individuos, su análisis deberá asumir esto de manera que se obtengan deberán ser interpretaciones de tipo hermenéutico.

\section{El futuro de la psicología social colectiva: la comunicación colectiva como proyecto e interpretación societal}

En este punto es necesario anotar que, para este presente de la disciplina, sus posibilidades de desarrollo se acrecientan por la coincidencia de intereses. Todo indica que las diferentes tradiciones, por caminos también diferentes se interesan en un sentido semejante en la comunicación y la cultura. Su desarrollo seguramente convergirá y divergirá en muchos aspectos, para beneficio de la propia disciplina. El periodo de crisis ha permitido entonces, que los contenidos temáticos y metodológicos de las diferentes aproximaciones sean reflexionados y, en algunos casos, transformados. Es evidente que esto tiene que suceder así, ya que la sociedad se transforma y las interpretaciones que hagamos en torno a ella tienen que crecer acordes a esto.

El interés de la psicología social colectiva se ubica en la comunicación social por ser este el proceso intersubjetivo que dibuja más fehacientemente nuestra realidad social. Decíamos que las colectividades se aprecian en sus objetivaciones y son estas las que conforman la cultura que es, a fin de cuentas, el interés de las interpretaciones derivadas de la comunicación intersubjetiva propias de la psicología social colectiva.

${ }^{12}$ El desvanecimiento de la psicología colectiva por tanto tiempo puede tener razones de "poder", sin embargo, esta interpretación fue continuada en terrenos más que académicos de tipo literario filosóficos. 


\section{Consideraciones finales}

El desarrollo histórico - conceptual del presente trabajo ha partido, más que de datos, de las construcciones conceptuales propias de las diferentes tradiciones incluidas en nuestra disciplina. Esta razón no ha sido casual, ya que se trata de una reinterpretación histórica a partir de lo que en nuestro presente se aprecia como la aproximación que más sentido da a nuestra realidad y a nuestra sociedad: la psicología colectiva.

\section{Referencias}

Allport, G. W. (1968): "The historical background of modern social psychology”, en: Lindzey \& Aronson (1968): The Handbook of Social Psychology, (2a Edición). Reading, Mass.: Addison Wesley, vol. 1.

Asch, S. (1952): Psicología Social. Buenos Aires: EUDEBA, 1962.

Berger, P. \& Luckman, T. (1983): La construcción social de la realidad. Buenos Aires: Amorrortu.

Doise, W. (1983): "Tensiones y explicaciones en psicología social experimental", Revista Mexicana de Sociología, México, Instituto de Investigaciones Sociales, UNAM, Abril - Junio, 1983.

Fernández, P. (1987): La intersubjetividad como fundamento de la psicología social. México: UNAM.

Festinger, L. (1957): A theory of cognitive dissonance. E.U.: Row Petterson.

Garai, L. \& Eros, F. (1978): "La teoría social de Marx y el concepto de hombre en psicología social",: Studia Psicología, 20, 1.

Habermas, J. (1982): Conocimiento e interés. Madrid: Taurus.

Heider, F. (1946): “Attitudes and Cognitive Organization”, Journal of Psychology, 27, 107-112.

Lewin, K. (1951): Field Theory in social sciences. New York: Harper.

Marx, K. (1844): Manuscritos Económico - Filosóficos. México: Ediciones de Cultura Popular, 1976.

Marx, K, (1845): La Ideología Alemana. México: Ediciones de Cultura Popular, 1976.

Mead, G.H. (1930): Espíritu, Persona y Sociedad: desde el punto de vista del conductismo social. Buenos Aires: Paidós, 1972.

Moscovici, S. (1972): "Society and theory in Social Psychology", en: H. Tajfel \& J. Israel (eds): The Context of social psychology, London: Academic Press, 1972.

Moscovici. S. (1981): La era de las multitudes. México: Fondo de Cultura Económica, 1984.

Moscovici, S. (1961): La psychanalyse, son image et son public, (2ª edición). París: PUF, 1976.

Munné, F. (1982): Psicología sociales marginadas: la línea de Marx en la Psicología Social. Barcelona: Hispano Europea.

Sherif, M. \& Sherif, C. (1969): Social Psychology. New York, Harper \& Row. 
Tajfel, H. (1981): Human Groups and Social Categories. London: Cambridge University Press.

Tajfel, H. (1972): “Introduction”, en: H. Tajfel \& J. Israel (eds): The Context of Social Psychology, London: Academic Press.

Zajonc, R.B. (s/f): La Psicología Social: Estudios Experimentales. Madrid: Marfil, 1967.

\section{Formato de citación}

Bautista, A. y Martínez, G. (2006). Esbozo histórico-conceptual de la psicología social comunicativa, desde un punto de vista comunicativo.. Athenea Digital, 10, 185-194. Disponible en http://antalya.uab.es/athenea/num10/BautistaM.pdf.

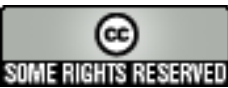

Este texto está protegido por una licencia Creative Commons.

Usted es libre de copiar, distribuir y comunicar públicamente la obra bajo las siguientes condiciones:

Reconocimiento: Debe reconocer y citar al autor original.

No comercial. No puede utilizar esta obra para fines comerciales.

Sin obras derivadas. No se puede alterar, transformar, o generar una obra derivada a partir de esta obra.

$\underline{\text { Resumen de licencia }}$

Texto completo de la licencia 